\title{
Numerical Investigation of the Role of Volumetric Transformation Strain on the Relaxation Stress and the Corresponding Hydrogen Interstitial Concentration in Niobium Matrix
}

\author{
Burak Bal ${ }^{1,2}$ \\ ${ }^{1}$ Department of Mechanical Engineering, Abdullah Gül University, 38080 Kayseri, Turkey \\ ${ }^{2}$ Department of Mechanical \& Aerospace Engineering, University of California, Los Angeles (UCLA), Los Angeles, CA 90095, USA
}

Correspondence should be addressed to Burak Bal; burak.bal@agu.edu.tr

Received 8 December 2016; Accepted 30 March 2017; Published 26 April 2017

Academic Editor: Carlo Santulli

Copyright (c) 2017 Burak Bal. This is an open access article distributed under the Creative Commons Attribution License, which permits unrestricted use, distribution, and reproduction in any medium, provided the original work is properly cited.

The effects of relaxation stress on the hydrogen concentration in Niobium- (Nb-) H media were investigated by iterative numerical modeling approach. To calculate the transformation strain, relaxation stress, and corresponding relaxed hydrogen concentration around an edge dislocation, a new third-order polynomial formulation was utilized in the model. With the aid of this polynomial, hydrogen induced relaxation stress never exceeds the dislocation stress, which indicates that the total stress field never turns to compressive state and diverges the results. The current model calculates the hydrogen concentration not only in the vicinity of an edge dislocation but also far away from the dislocation. Furthermore, the effect of relaxation stress on the interaction energy was also captured in the model. Overall, the current findings shed light on the complicated hydrogen embrittlement mechanisms of metallic materials by demonstrating that hydrogen induced relaxation has a significant effect on the hydrogen atom concentration and the interaction energy between the existing internal stress field and the solute hydrogen atom.

\section{Introduction}

Hydrogen embrittlement (HE) is a severe material degradation mechanism which deteriorates the mechanical properties of materials [1-3]. Once hydrogen diffuses into a host matrix, materials usually fail at low stress levels when compared to their hydrogen free counterparts $[4,5]$. Also, the tendency to HE is correlated with the strength of the material; that is, the higher the strength, the higher the susceptibility to HE [6, 7]. In order to use hydrogen as a clean energy source over fossil fuels, to utilize high strength steels in automotive and defense industries and to design safe hydrogen storage materials, the mechanism of hydrogen embrittlement should be understood thoroughly. Many researchers have focused on the degradation of tensile properties [8], fracture toughness [6], and fatigue crack rate of the materials [9]. Moreover, effects of alloying elements [10, 11], hydrogen content [4, 12], and grain size [13] on HE behavior of materials have been reported previously. However, despite the fact that HE has been investigated extensively since 1875 [14], the exact mechanism of $\mathrm{HE}$ remains unclear.

Several candidate mechanisms have been suggested by different researchers to explain the HE phenomenon in materials and three of them acquired much attention, namely, hydride formation and cleavage [15-17], hydrogen enhanced decohesion (HEDE) $[18,19]$, and hydrogen enhanced localized plasticity (HELP) $[20,21]$. The underlying principle of the hydride formation and cleavage mechanism is that hydride formation creates a local stress concentration and when the material cannot accommodate it plastically, sudden brittle fracture occurs [17]. Hydride formation and cleavage mechanism is the active mechanism for systems in which hydrides are either stable or can be stabilized with hydrostatic stress, such as zirconium ( $\mathrm{Zr}$ ), titanium (Ti), and Niobium (Nb) [22]. The second mechanism, HEDE, states that hydrogen weakens the interatomic bonds at the crack 
tip and causes brittle or transgranular fracture [23]. The last mechanism, HELP, states that hydrogen in the host matrix increases the mobility of dislocations by reducing the barriers against dislocation motion or promotes the nucleation of dislocations and causes local instability in the plastic flow. HELP mechanism, which is based on both experimental observations and theoretical validations, is the most widely used HE mechanism. However, there is no single applicable HE mechanism to all materials: instead, each HE mechanism has different roles depending on the material and external factors. Moreover, coexistence of HE mechanisms, that is, HELP + HEDE, was observed both experimentally [24-26] and theoretically $[27,28]$ depending on the local hydrogen concentration and microstructure. Therefore, reduction of both effects would be needed to obtain higher resistance against HE. Hydrogen transportation process plays a crucial role in deciding which HE mechanisms are active during the deformation.

Once hydrogen diffuses into the material, it can locate either to normal interstitial lattice sites (NILS) or to reversible trap sites, depending on the trap binding energy and the occupancy of lattice/trap sites [29]. Specifically, hydrogen atoms tend to diffuse into the site which has greater binding energy [30]. Several microstructural heterogeneities, dislocations, vacancies, voids, inclusions, grain boundaries, and impurities can trap hydrogen and it has been demonstrated that hydrogen trapping plays a significant role for HE. Therefore, modeling the hydrogen distribution around a dislocation is of utmost importance in order to understand the underlying principle of HE.

Many of the previous studies have reported several hydrogen concentration distribution models [31-34]. Consequently, it has been well known that accurate modeling of hydrogen concentration distribution both in the vicinity of a dislocation and remote from the dislocation should be carried out while investigating HE. However, there are only a limited number of studies investigating the effect of transformation on the relaxation stress and the corresponding hydrogen concentration. In particular, once hydrogen diffuses into the lattice and expands the lattice, the internal stress field around the hydrogen atoms changes during expansion and triggers volumetric strain and corresponding relaxation [32]. Even though different transformation strain formulations are available in the current literature, such as fourth-order [32], first-order [35], logarithmic [36], and proportional [33], to the best of the author's knowledge, a third-order polynomial formulation, which ensures faster decrease of the relaxation stress than the dislocation stress, has not been forwarded yet.

In this study, third-order polynomial formulation was proposed to calculate the hydrogen induced volumetric strain and the corresponding transformation strain and relaxation stress of hydrogen atoms introduced to the host matrix. With the aid of relaxation stress, hydrogen concentration distribution both in the vicinity of a dislocation and remote from the dislocation was calculated. Current results clearly show that the relaxation stress due to hydrogen is always less than the dislocation stress and demonstrate the effect of relaxation on the hydrogen distribution. The current model was applied to the Niobium- (Nb-) H media.

\section{Theory and Calculations}

Hydrostatic stress dependent hydrogen diffusion can be defined as [37]

$$
\frac{\partial C}{\partial t}=D_{H} \nabla^{2} C-\frac{D_{H} V_{H} \nabla\left(C \nabla \sigma_{h}\right)}{R T},
$$

where $C$ is the local hydrogen concentration, $t$ is the time, $D_{H}$ is the diffusion coefficient of hydrogen, $V_{H}$ is the partial molar volume of hydrogen, $\sigma_{h}$ is the hydrostatic stress $\left((1 / 3) \sigma_{i i}\right)$ in the neck of the specimen, $R$ is the gas constant, and $T$ is the absolute temperature. For the 2D axisymmetric model, (1) can be expressed as [37]

$$
\begin{gathered}
\frac{\partial C}{\partial t}=D_{H}\left(\frac{\partial^{2} C}{\partial r^{2}}+\frac{1}{r} \frac{\partial C}{\partial r}+\frac{\partial^{2} C}{\partial z^{2}}\right) \\
-\frac{D_{H} V_{H}}{R T}\left[\left(\frac{\partial C}{\partial r} \frac{\partial \sigma_{h}}{\partial r}+\frac{\partial C}{\partial z} \frac{\partial \sigma_{h}}{\partial z}\right)\right. \\
\left.+C\left(\frac{\partial^{2} \sigma_{h}}{\partial r^{2}}+\frac{1}{r} \frac{\partial \sigma_{h}}{\partial r}+\frac{\partial^{2} \sigma_{h}}{\partial z^{2}}\right)\right] .
\end{gathered}
$$

After neglecting hydrogen release from the specimen surface, which satisfies zero flux boundary conditions and assuming uniform initial hydrogen concentration, the stress distribution and the corresponding hydrogen diffusion can be calculated through finite difference method. The reader is referred to [37] for the details of the calculation method. It is wellknown that a tension triaxial stress decreases and a compression triaxial stress increases the chemical potential of hydrogen, and the hydrogen interstitial concentration around a dislocation is given by [37-40]

$$
C=\frac{C_{0}}{\exp \left(W_{\text {int }} / R T\right)},
$$

where $C_{0}$ is the initial hydrogen concentration in a stress free region and $W_{\text {int }}$ is an interaction energy. Once hydrogen diffuses into the matrix, it expands the lattice and causes the planar interaction energy between the hydrogen atom and the existing internal stress field, given by [38]

$$
W_{\text {int }}=-\sigma_{h} V_{H} .
$$

The pointwise hydrostatic stress around an edge dislocation can be calculated as [32]

$$
\sigma_{h}=-\frac{G}{2 \pi(1-\vartheta)}\left(\frac{b \sin \theta}{r}\right) .
$$

The terms $r$ and $\theta$ define the cylindrical coordinate system with the positive edge dislocation at the origin, and $G$ is shear modulus, $\vartheta$ is Poisson's ratio, and $b$ is Burgers vector. Using the number of solvent lattice atoms per unit lattice volume, $N_{L}$, the number of hydrogen atoms per solvent atom can be calculated as [33]

$$
C=c N_{L}
$$


TABLE 1: The parameters and constants utilized in the current model for the $\mathrm{Nb}-\mathrm{H}$ system.

\begin{tabular}{lccccccc}
\hline$V_{H}\left(\mathrm{~m}^{3} \mathrm{~mol}^{-1}\right)[33]$ & $V_{M}\left(\mathrm{~m}^{3} \mathrm{~mol}^{-1}\right)[33]$ & $b(\mathrm{~nm})[33]$ & $\vartheta[33]$ & $N_{L}[33]$ & $R(\mathrm{~J} / \mathrm{mol})$ & $G(\mathrm{GPa})[33]$ & $T(\mathrm{~K})$ \\
\hline $1.88 \times 10^{-6}$ & $10.8 \times 10^{-6}$ & 0.285 & 0.415 & $0.55 \times 10^{29}$ & 8.314 & 30.8 & 298 \\
\hline
\end{tabular}

Solute hydrogen atom in a host matrix induces dilatation strain as a function of the local hydrogen concentration and the partial molar volume, given by

$$
e^{H}=\frac{\left(c^{2}-c^{3}\right) V_{H}}{V_{M}}
$$

where $V_{H}$ is the molar volume of the host metal. Transformation strain varies pointwise and can be calculated as [35]

$$
\varepsilon_{i j}^{H}=\frac{1+\vartheta}{9(1-\vartheta)} e^{H} \delta_{i j},
$$

where $\delta_{i j}$ is the Kroneckar delta. Hydrogen induced relaxation stress in the host matrix is proportional to the pointwise transformation strain, given by [32]

$$
\sigma_{i j}^{H}=-C_{i j k l} \varepsilon_{i j}^{H},
$$

where $\sigma_{i j}^{H}$ is the pointwise components of the elastic stress tensor due to hydrogen and $C_{i j k l}$ is the linear elastic modulus of the system. The mechanical equilibrium of the system in the absence of body forces is satisfied through [33]

$$
\int \sigma_{i j} \delta \varepsilon_{i j}^{H} d V=0
$$

where $\delta$ is an arbitrary virtual variation of the quantity it precedes and $V$ is the volume of the system.

The shear stress imposed on a positive edge dislocation due to the solute hydrogen can be calculated as [33]

$$
d \tau_{H}=n d S\left(-\frac{G \Delta a}{\pi r^{2}}\right) \sin 2 \theta
$$

where $n$ is the outward unit normal vector and $\Delta a$ is the strength of the dilatation line. After integration over the entire area $S$, hydrogen induced net shear stress, $\tau_{H}$, which can be calculated through (12). The reader is referred to $[33,41]$ for the details of the calculation method.

$$
\tau_{H}=f\left(G, V_{H}\right) \int_{0}^{2 \pi} \int_{r_{i}}^{r_{o}} C(r, \varnothing) \frac{\sin 2 \theta}{r} d r d \theta,
$$

where $r_{i}$ and $r_{o}$ are the inner and outer cut-off radii of the $H$ atmosphere surrounding the edge dislocation. Since there is a singularity at the dislocation core, inner cut-off radii should be greater than 0 . The parameters and constants utilized in the current model for the $\mathrm{Nb}-\mathrm{H}$ system are presented in Table 1.

\section{Results and Discussion}

An iterative numerical model was proposed to calculate the hydrogen induced stress field and the corresponding hydrogen distribution around an edge dislocation. Even though

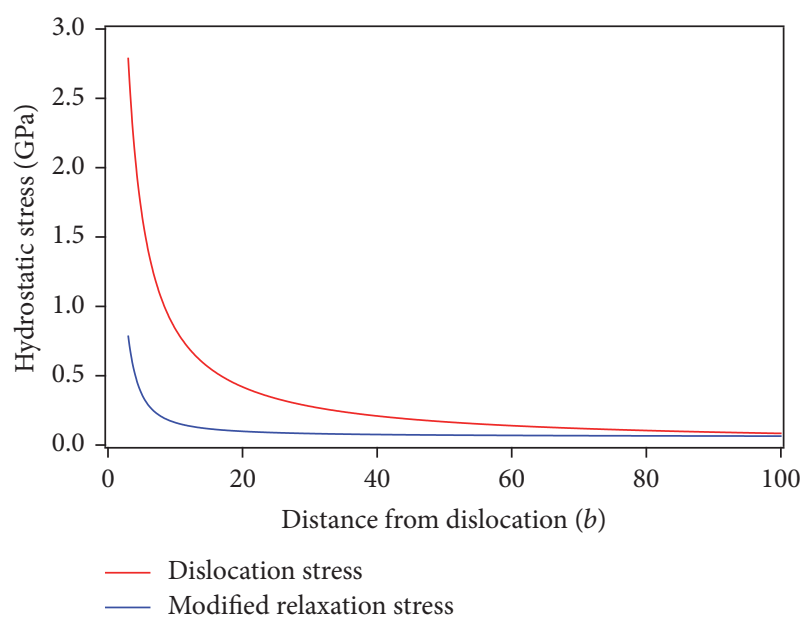

FIgURE 1: Hydrogen induced modified relaxation stress and hydrostatic stress as a function of distance from the core of an edge dislocation.

$\mathrm{Nb}-\mathrm{H}$ media were selected in the current study as a candidate system since it is well-studied and all related parameters are available in the literature, the model is applicable to all metallic materials. In calculating the hydrogen concentration profiles, the initial uniform number of hydrogen atoms per solvent atom, $c_{0}$, was taken as 0.1 in accordance with previous studies $[32,33,35,42]$ since it is a critical case to simulate the hydrostatic stress and the hydrogen concentration. The relaxation stress due to the introduction of hydrogen is always less than the magnitude of hydrostatic stress due to the dislocation (Figure 1). This result is in good agreement with previous studies $[32,33]$. It should be noted that both dislocation and relaxation hydrostatic stresses at the dislocation core are infinite and they decrease dramatically after a few Burgers vectors from an edge dislocation. Moreover, in Figure 1 the absolute values of relaxation stress were displayed since it is originally negative. The effect of modified relaxation stress is much more pronounced in the vicinity of dislocation $(\sim 800 \mathrm{MPa})$ and its effect decreases along the distance. Similar behavior was also observed in other studies $[31,32]$. Furthermore, the difference between dislocation stress and modified relaxation stress is also greater at the regions close to the dislocation and it becomes negligible after a certain distance from the dislocation. However, hydrogen induced relaxation stress never exceeds the dislocation stress, which indicates that the total stress field never turns to compressive state.

Figure 2 shows the contours of normalized initial hydrogen concentration around an edge dislocation in $\mathrm{Nb}$ matrix. Note that the sign of the Burgers vector of dislocation is positive. Relaxation effect was not considered while calculating the initial hydrogen concentration. In particular, hydrostatic 


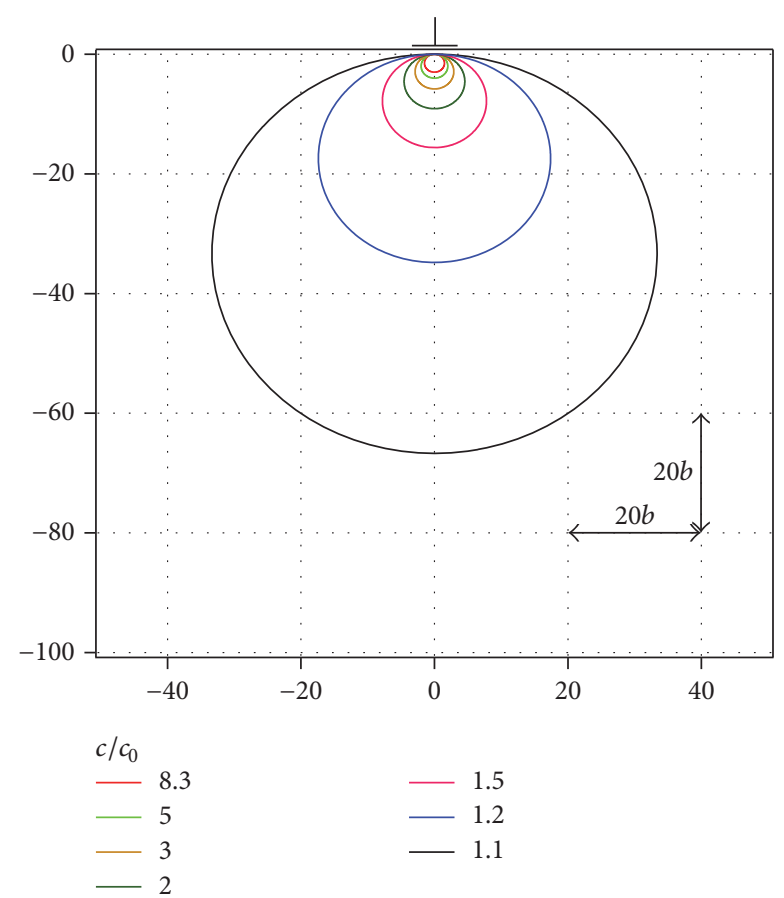

FIGURE 2: Contours of normalized initial hydrogen concentration around an edge dislocation in $\mathrm{Nb}$ matrix.

stress distribution around the defined dislocation region was calculated to find the interaction energy between the solute hydrogen atom and the existing internal stress field. Using the calculated interaction energy and assuming nominal hydrogen concentration $c_{0}=0.1$, corresponding numbers of hydrogen atoms per unit volume and per host atom were modeled. Hydrogen distribution around the dislocation was normalized with respect to the initial number of hydrogen atoms per solvent atom. Hydrogen concentration was modeled not only in the vicinity of an edge dislocation but also far away from the dislocation (Figure 3). The hydrogen distribution around an edge dislocation is symmetric with respect to the dislocation plane mainly due to the symmetry in the hydrostatic stress field of dislocation. Similar hydrogen distribution behavior was also reported in previous studies $[32,33,42]$. It was also reported that the hydrogen segregation ratio was enhanced at the tip of pile-up due to cumulative hydrostatic stress fields of dislocations [42].

Figure 3 shows the contours of normalized relaxed hydrogen concentration around an edge dislocation in $\mathrm{Nb}$ matrix. Note that the sign of the Burgers vector of dislocation is positive. Specifically, dilatation strain around the dislocation region was calculated based on third-order polynomial formulation and corresponding transformation and relaxation stress values were calculated by iterative method until the error converged to $10^{-5}$. Hydrogen induced relaxation stress was implemented into the hydrostatic stress distribution due to dislocation to calculate the modified interaction energy and the corresponding relaxed hydrogen concentration, which were then normalized with respect to the initial hydrogen atoms per solvent atom. Note that while calculating

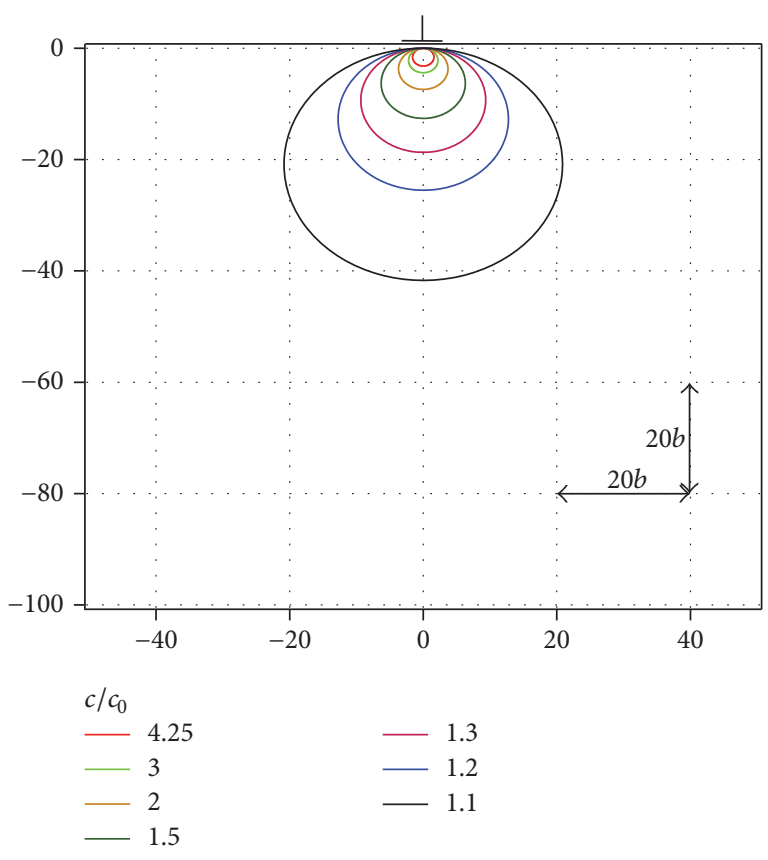

FIGURE 3: Contours of normalized relaxed hydrogen concentration around an edge dislocation in $\mathrm{Nb}$ matrix.

the relaxation stress, the effect of uniform strain contribution was disregarded and only pointwise transformation strain was taken into account since the elastic modulus of $\mathrm{Nb}$ is high enough to induce much more hydrogen induced transformation strain than uniform strain. Using iterative approach, relaxed hydrogen concentration both around an edge dislocation and far away from the dislocation was captured. Specifically, no normalized high hydrogen concentration greater than 5 was allowed under relaxation effect all around the dislocation region. In the vicinity of an edge dislocation, solute normalized hydrogen concentration was reduced to half of its initial value with the aid of hydrogen induced relaxation stress. Furthermore, as the distance from the core of an edge dislocation increases, the difference between the relaxed hydrogen concentration and the initial hydrogen concentration becomes smaller so that the effect of relaxation stress on solute hydrogen concentration decreases since the solution becomes more dilute with distance. The results correspond well with the previous studies on $\mathrm{Nb}-\mathrm{H}$ media, in which different dilatation and transformation strain modeling were carried out [30, 32]. The interaction between hydrogen atom and dislocations can change the mechanical properties of materials dramatically. Therefore it is significant to model the hydrogen concentration (Figures 2 and 3 ) in the material in order to determine these interactions and corresponding results. It is known that hydrogen atom shields the elastic interactions between dislocations and obstacles and enhances the dislocation mobility (HELP mechanism). Inhomogeneous distribution of hydrogen concentration around dislocations can also start dislocation avalanches and mobile dislocations can sweep hydrogen atoms around dislocations (Figures 2 and 3 ) to the grain boundaries during deformation. 


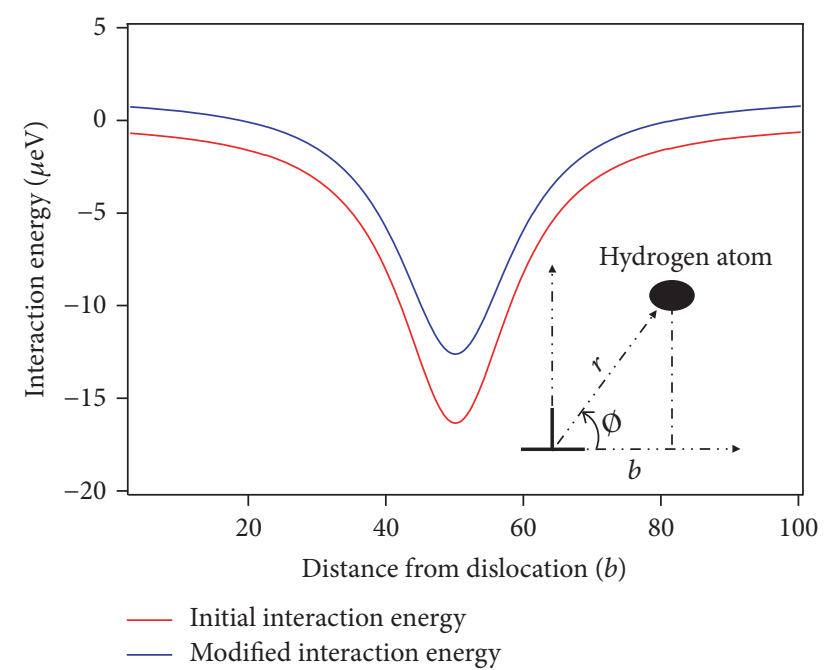

Figure 4: Modified and initial interaction energies between the existing internal stress field and solute hydrogen atom as a function of distance from the core of an edge dislocation.

Once dislocations reach the grain boundary they can distort it and create surface steps, which may turn into grain boundary cracks. Transported hydrogen can locate to these steps and presence of hydrogen on grain boundary reduces cohesive energy, which depends on the segregated hydrogen content, and enhance brittle intergranular fracture which is more pronounced at higher local hydrogen concentrations. (HEDE mechanism and the proposed mechanism in the author's former paper [7]). Moreover coexistence of different HE mechanisms (e.g., HELP + HEDE) may also be observed depending on the local hydrogen concentration.

Figure 4 shows the initial and modified interaction energies between the existing internal stress field and the solute hydrogen atom as a function of distance from the core of an edge dislocation. In both cases, the magnitude of interaction energy first increases and then decreases and becomes negligible after a certain distance from an edge dislocation. This behavior is mainly because when the dislocation approaches the solute hydrogen, the distance between the dislocation and the hydrogen atom becomes smaller, and the interaction energy between an existing internal stress field and the solute hydrogen atom increases. Consequently, when the dislocation passes the solute hydrogen atom and glides along the slip plane, the distance between the dislocation and hydrogen atom becomes larger and the interaction energy between an existing internal stress field and the solute hydrogen atom decreases. The interaction energy becomes nearly zero after a certain distance, which results in no further expansion in the lattice due to hydrogen. The schematic representation of solute hydrogen and dislocation-hydrogen system is given as an inset in Figure 4. When the relaxation stress due to hydrogen atom was taken into account, the hydrostatic stress and the corresponding interaction energy values decrease. Therefore, the magnitude of initial interaction energy becomes greater than the magnitude of relaxed interaction energy due to the introduction of solute hydrogen atom into the lattice.
A similar interaction energy trend due to solute hydrogen was also observed in the previous studies $[33,43]$.

\section{Conclusion}

In this study, the effects of relaxation stress on the hydrogen concentration in $\mathrm{Nb}-\mathrm{H}$ media were investigated by iterative numerical modeling approach. A unique third-order polynomial formulation was used to calculate the transformation strain, the corresponding relaxation stress, and the relaxed hydrogen concentration around an edge dislocation. From the work presented herein the following conclusions can be drawn:

(1) Hydrogen induced relaxation stress, calculated based on the third-order polynomial formulation, never exceeds the dislocation stress throughout the whole dislocation region. Hence, total stress field never turns to compressive state.

(2) The hydrogen induced relaxation stress is dominant in the vicinity of an edge dislocation, whereas its effect decreases with increasing distance from the core of the dislocation. Specifically, hydrogen relaxation stress reduces the corresponding hydrogen concentration around $50 \%$ in the vicinity of an edge dislocation. Consequently, the effect of relaxation stress on solute hydrogen concentration decreases since the solution becomes more dilute with increasing distance.

(3) As the dislocation glides on the slip system, the magnitude of the interaction energy between the existing internal stress field and the solute hydrogen atom increases until the distance between the dislocation and the solute hydrogen reaches a minimum. Further dislocation glide decreases the magnitude of the interaction energy and at the saturation it becomes nearly zero, which results in no further expansion in the lattice due to hydrogen.

\section{Conflicts of Interest}

The author declares that there are no conflicts of interest regarding the publication of this paper.

\section{Acknowledgments}

B. Bal acknowledges the financial support by the Scientific and Technological Research Council of Turkey (TÜBİTAK) BIDEB-2219 Postdoctoral Research program under Project no. 1059B191501308. The author would also like to acknowledge Professor Nasr Ghoniem for taking part in the discussion during the preparation of research proposal.

\section{References}

[1] G. P. Tiwari, A. Bose, J. K. Chakravartty et al., "A study of internal hydrogen embrittlement of steels," Materials Science and Engineering A, vol. 286, no. 2, pp. 269-281, 2000. 
[2] Y. Liang and P. Sofronis, “Toward a phenomenological description of hydrogen-induced decohesion at particle/matrix interfaces," Journal of the Mechanics and Physics of Solids, vol. 51, no. 8, pp. 1509-1531, 2003.

[3] W.-S. Ko, J. B. Jeon, J.-H. Shim, and B.-J. Lee, "Origin of hydrogen embrittlement in vanadium-based hydrogen separation membranes," International Journal of Hydrogen Energy, vol. 37, no. 18, pp. 13583-13593, 2012.

[4] Y. Qi, H. Luo, S. Zheng, C. Chen, and D. Wang, "Effect of immersion time on the hydrogen content and tensile properties of A350LF2 steel exposed to hydrogen sulphide environments," Corrosion Science, vol. 69, pp. 164-174, 2013.

[5] D. M. Symons, "The effect of carbide precipitation on the hydrogen-enhanced fracture behavior of alloy 690," Metallurgical and Materials Transactions A, vol. 29, no. 4, pp. 1265-1277, 1998.

[6] M. Nagumo, M. Nakamura, and K. Takai, "Hydrogen thermal desorption relevant to delayed-fracture susceptibility of highstrength steels," Metallurgical and Materials Transactions A, vol. 32, no. 2, pp. 339-347, 2001.

[7] B. Bal, M. Koyama, G. Gerstein, H. J. Maier, and K. Tsuzaki, "Effect of strain rate on hydrogen embrittlement susceptibility of twinning-induced plasticity steel pre-charged with highpressure hydrogen gas," International Journal of Hydrogen Energy, vol. 41, no. 34, pp. 15362-15372, 2016.

[8] B. Bal, I. Sahin, A. Uzun, and D. Canadinc, "A new venue toward predicting the role of hydrogen embrittlement on metallic materials," Metallurgical and Materials Transactions A, vol. 47, no. 11, pp. 5409-5422, 2016.

[9] Y. Murakami and S. Matsuoka, "Effect of hydrogen on fatigue crack growth of metals," Engineering Fracture Mechanics, vol. 77, no. 11, pp. 1926-1940, 2010.

[10] J. Zhao, Z. Jiang, and C. S. Lee, "Effects of tungsten on the hydrogen embrittlement behaviour of microalloyed steels," Corrosion Science, vol. 82, pp. 380-391, 2014.

[11] T. Dieudonné, L. Marchetti, M. Wery et al., "Role of copper and aluminum additions on the hydrogen embrittlement susceptibility of austenitic Fe-Mn-C TWIP steels," Corrosion Science, vol. 82, pp. 218-226, 2014.

[12] M. Koyama, E. Akiyama, and K. Tsuzaki, "Effect of hydrogen content on the embrittlement in a Fe-Mn-C twinning-induced plasticity steel," Corrosion Science, vol. 59, pp. 277-281, 2012.

[13] Y. Bai, Y. Momotani, M. C. Chen, A. Shibata, and N. Tsuji, "Effect of grain refinement on hydrogen embrittlement behaviors of high-Mn TWIP steel," Materials Science and Engineering A, vol. 651, pp. 935-944, 2016.

[14] W. H. Johnson, "On some remarkable changes produced in iron and steel by the action of hydrogen and acids," Proceedings of the Royal Society of London, vol. 23, pp. 168-179, 1875.

[15] S. Gahr, M. L. Grossbeck, and H. K. Birnbaum, "Hydrogen embrittlement of $\mathrm{Nb} \mathrm{I}-$ macroscopic behavior at low temperatures," Acta Metallurgica, vol. 25, no. 2, pp. 125-134, 1977.

[16] D. S. Shih, I. M. Robertson, and H. K. Birnbaum, "Hydrogen embrittlement of $\alpha$ titanium: in situ tem studies," Acta Metallurgica, vol. 36, no. 1, pp. 111-124, 1988.

[17] J. Lufrano, P. Sofronis, and H. K. Birnbaum, "Modeling of hydrogen transport and elastically accommodated hydride formation near a crack tip," Journal of the Mechanics and Physics of Solids, vol. 44, no. 2, pp. 179-205, 1996.

[18] A. Troiano, "The role of hydrogen and other interstitials in the mechanical behavior of metals," Transactions of ASM, vol. 52, pp. 54-80, 1960.
[19] R. A. Oriani and P. H. Josephic, "Equilibrium and kinetic studies of the hydrogen-assisted cracking of steel," Acta Metallurgica, vol. 25, no. 9, pp. 979-988, 1977.

[20] C. D. Beachem, "A new model for hydrogen-assisted cracking (hydrogen 'embrittlement')," Metallurgical and Materials Transactions, vol. 3, pp. 437-451, 1972.

[21] H. K. Birnbaum and P. Sofronis, "Hydrogen-enhanced localized plasticity-a mechanism for hydrogen-related fracture," Materials Science and Engineering A, vol. 176, no. 1-2, pp. 191-202, 1994.

[22] W. Qin, J. A. Szpunar, and J. Kozinski, "Hydride-induced degradation of hoop ductility in textured zirconium-alloy tubes: a theoretical analysis," Acta Materialia, vol. 60, no. 12, pp. 48454855, 2012.

[23] M. Djukic, V. S. Zeravcic, G. Bakic, A. Sedmak, and B. Rajicic, "Hydrogen embrittlement of low carbon structural steel," Procedia Materials Science, vol. 3, pp. 1167-1172, 2014.

[24] M. B. Djukic, V. Sijacki Zeravcic, G. M. Bakic, A. Sedmak, and B. Rajicic, "Hydrogen damage of steels: a case study and hydrogen embrittlement model," Engineering Failure Analysis, vol. 58, pp. 485-498, 2015.

[25] M. B. Djukic, G. M. Bakic, V. S. Zeravcic, A. Sedmak, and B. Rajicic, "Hydrogen embrittlement of industrial components: prediction, prevention, and models," Corrosion, vol. 72, no. 7, pp. 943-961, 2016.

[26] M. Koyama, C. C. Tasan, E. Akiyama, K. Tsuzaki, and D. Raabe, "Hydrogen-assisted decohesion and localized plasticity in dualphase steel," Acta Materialia, vol. 70, pp. 174-187, 2014.

[27] P. Novak, R. Yuan, B. Somerday, P. Sofronis, and R. Ritchie, "A statistical, physical-based, micro-mechanical model of hydrogen-induced intergranular fracture in steel," Journal of the Mechanics and Physics of Solids, vol. 58, no. 2, pp. 206-226, 2010.

[28] R. Matsumoto, S. Seki, S. Taketomi, and N. Miyazaki, "Hydrogen-related phenomena due to decreases in lattice defect energies-molecular dynamics simulations using the embedded atom method potential with pseudo-hydrogen effects," Computational Materials Science, vol. 92, pp. 362-371, 2014.

[29] A. Taha and P. Sofronis, "A micromechanics approach to the study of hydrogen transport and embrittlement," Engineering Fracture Mechanics, vol. 68, no. 6, pp. 803-837, 2001.

[30] Y. Liang, P. Sofronis, and R. Dodds, "Interaction of hydrogen with crack-tip plasticity: effects of constraint on void growth," Materials Science and Engineering. A, vol. 366, no. 2, pp. 397411, 2004.

[31] S. Wang, N. Hashimoto, Y. Wang, and S. Ohnuki, "Activation volume and density of mobile dislocations in hydrogen-charged iron," Acta Materialia, vol. 61, no. 13, pp. 4734-4742, 2013.

[32] M. C. Uslu and D. Canadinc, "Modeling the role of hydrogen interstitial concentration on internal stress fields in iron matrix," Journal of Materials Science, vol. 45, no. 6, pp. 1683-1687, 2010.

[33] P. Sofronis and H. K. Birnbaum, "Mechanics of the hydrogendashdislocationdashimpurity interactions-I. Increasing shear modulus," Journal of the Mechanics and Physics of Solids, vol. 43, no. 1, pp. 49-90, 1995.

[34] P. Sofronis, Y. Liang, and N. Aravas, "Hydrogen induced shear localization of the plastic flow in metals and alloys," European Journal of Mechanics-A/Solids, vol. 20, no. 6, pp. 857-872, 2001.

[35] D. Delafosse and T. Magnin, "Hydrogen induced plasticity in stress corrosion cracking of engineering systems," Engineering Fracture Mechanics, vol. 68, no. 6, pp. 693-729, 2001.

[36] A. H. M. Krom, R. W. J. Koers, and A. Bakker, "Hydrogen transport near a blunting crack tip," Journal of the Mechanics and Physics of Solids, vol. 47, no. 4, pp. 971-992, 1999. 
[37] M. Wang, E. Akiyama, and K. Tsuzaki, "Crosshead speed dependence of the notch tensile strength of a high strength steel in the presence of hydrogen," Scripta Materialia, vol. 53, no. 6, pp. 713-718, 2005.

[38] B. Strnadel, "Failure of steels caused by hydrogen induced microcracking," Engineering Fracture Mechanics, vol. 61, no. 3-4, pp. 299-310, 1998.

[39] J. P. Hirth and B. Carnahan, "Hydrogen adsorption at dislocations and cracks in Fe," Acta Metallurgica, vol. 26, no. 12, pp. 1795-1803, 1978.

[40] A. H. Cottrell and M. A. Jaswon, "Distribution of solute atoms round a slow dislocation," Proceedings of the Royal Society A: Mathematical, Physical and Engineering Sciences, vol. 199, no. 1056, pp. 104-114, 1949.

[41] B. Bal, B. Gumus, and D. Canadinc, "Incorporation of dynamic strain aging into a viscoplastic self-consistent model for predicting the negative strain rate sensitivity of hadfield steel," Journal of Engineering Materials and Technology, vol. 138, no. 3, Article ID 031012, 8 pages, 2016.

[42] J. P. Chateau, D. Delafosse, and T. Magnin, "Numerical simulations of hydrogen-dislocation interactions in fcc stainless steels. Part II: hydrogen effects on crack tip plasticity at a stress corrosion crack," Acta Materialia, vol. 50, no. 6, pp. 1523-1538, 2002.

[43] F. C. Larché and J. W. Cahn, "Overview no. 41 The interactions of composition and stress in crystalline solids," Acta Metallurgica, vol. 33, no. 3, pp. 331-357, 1985. 

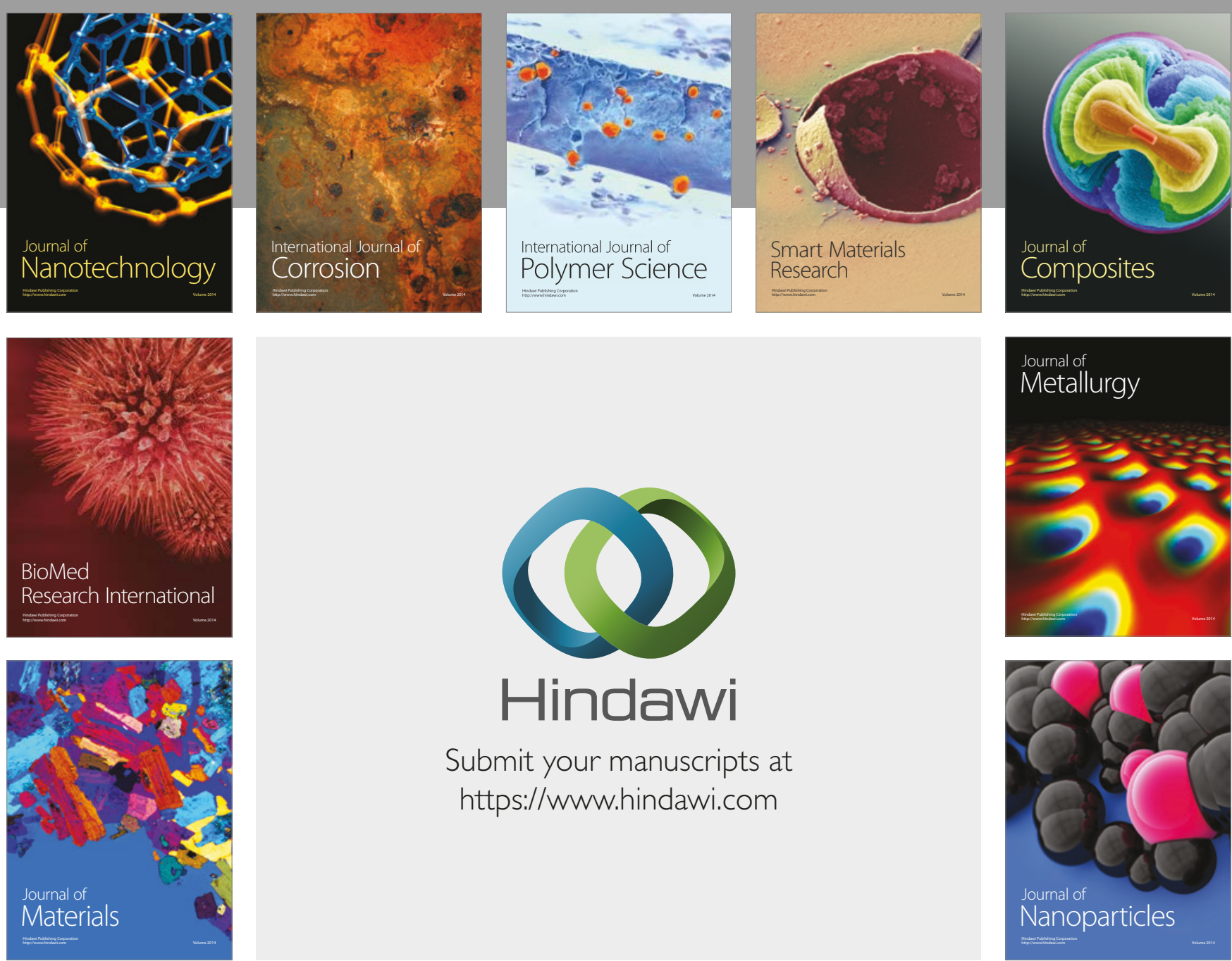

\section{Hindawi}

Submit your manuscripts at

https://www.hindawi.com
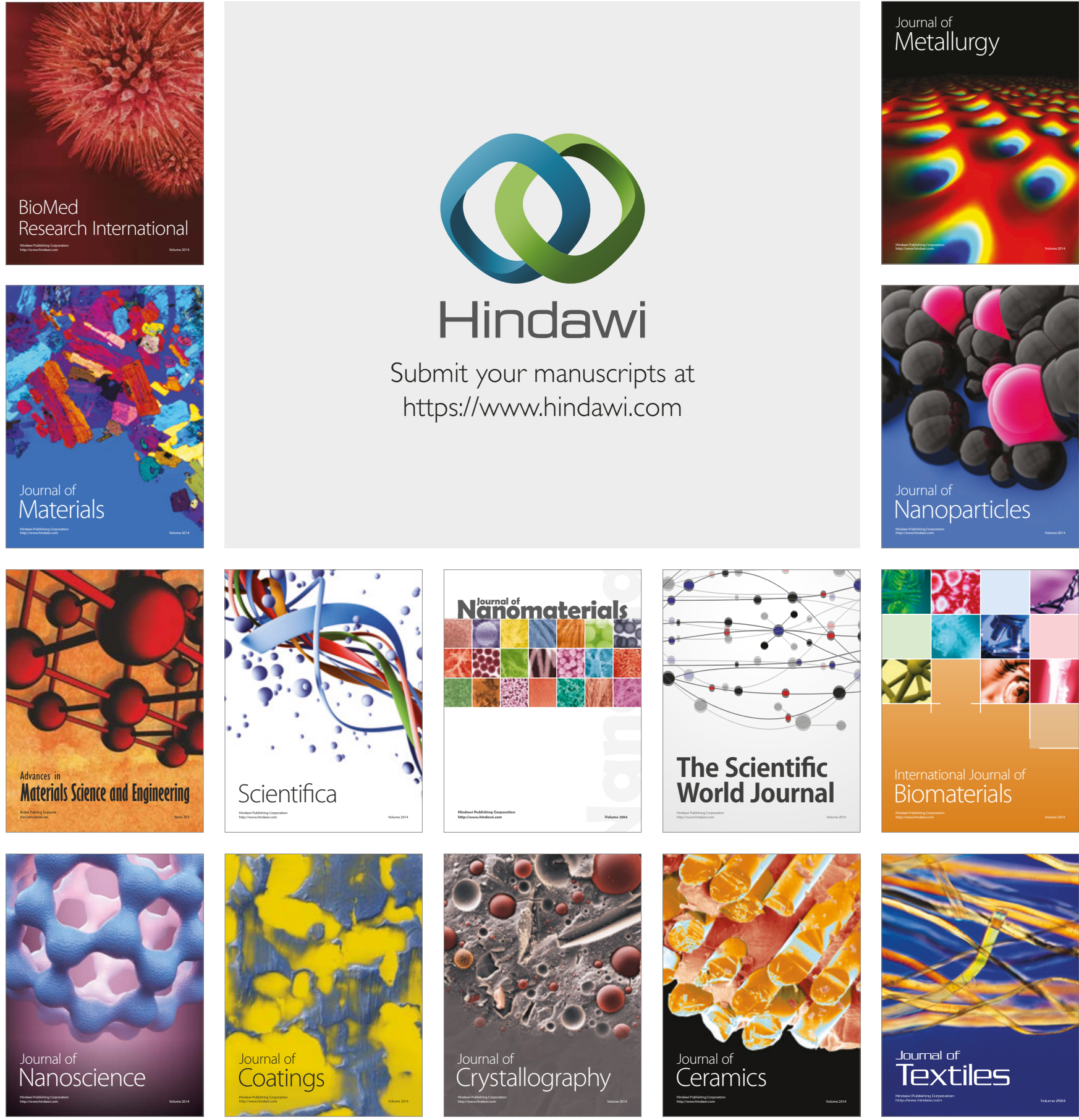

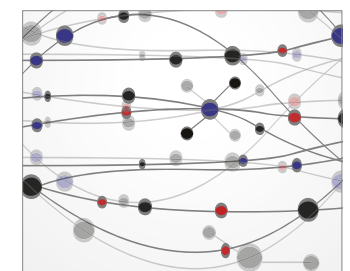

The Scientific World Journal
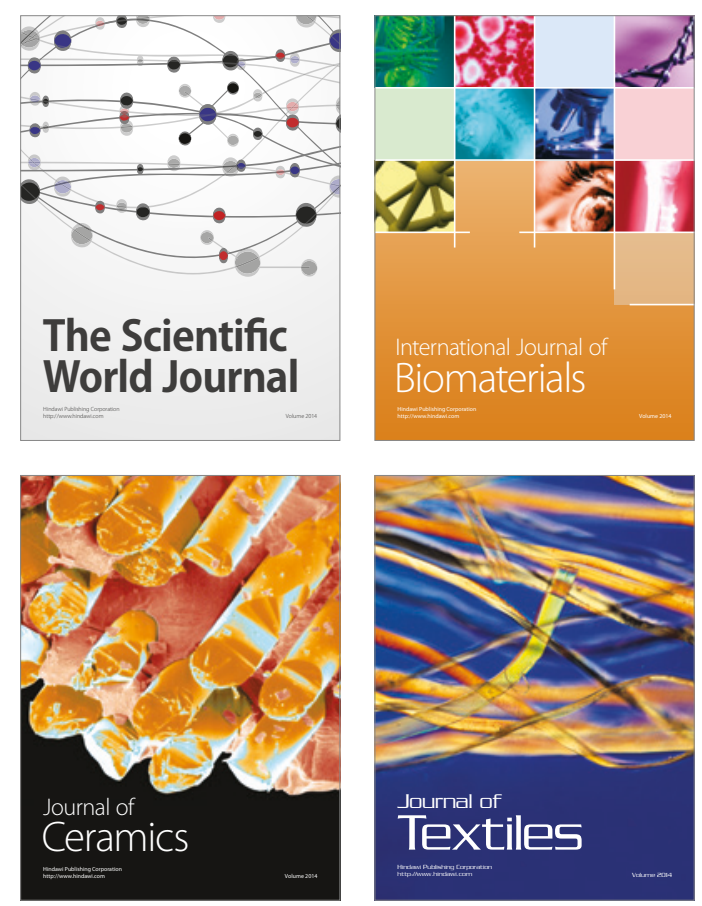